\title{
Retraction Note: Selected TLR7/8 agonist and type I interferon (IFN- $a$ ) cooperatively redefine the microglia transcriptome
}

\author{
Mst Reshma Khatun ${ }^{1} \cdot$ Sarder Arifuzzaman ${ }^{2}$ (1)
}

Published online: 15 June 2020

C) Springer Nature Switzerland AG 2020

\section{Retraction Note: Inflammopharmacology https://doi.org/10.1007/s10787-019-00610 $-88$}

The authors have retracted this article https://doi. org/10.1007/s10787-019-00610-8 because, since publication, it has become apparent that there are major errors in the qRT-PCR data presented in Figures 1 and 7.

Sarder Arifuzzaman agrees to this retraction/Mst Reshma Khatun has not responded to any correspondence from the editor/publisher about this retraction.

[1] Khatun, M.R. \& Arifuzzaman, S. Inflammopharmacol (2019). https://doi.org/10.1007/s10787-019-00610-8

The original article can be found online at https://doi.org/10.1007/ s10787-019-00610-8.

Sarder Arifuzzaman

arifuzzaman@cau.ac.kr

Mst Reshma Khatun

reshma@ajou.ac.kr

1 Department of Biomedical Science, Ajou University, Suwon, Gyeonggi-do 16499, Republic of Korea

2 Department of Animal Science and Technology, Chung-Ang University, Anseong, Gyeonggi-do 17546, Republic of Korea 